\title{
Composite Liquid Marbles as a Macroscopic Model System Representing Shedding of Enveloped Viruses via a Budding Mechanism
}

Pritam Kumar Roy ${ }^{\mathrm{a}}$, Bernard P. Binks ${ }^{\mathrm{b}}$, Syuji Fujii ${ }^{\mathrm{c}, \mathrm{d}}$, Shraga Shoval ${ }^{\mathrm{e}}$, Edward Bormashenko,

${ }^{a}$ Chemical Engineering Department, Engineering Faculty, Ariel University, P.O.B. 3, 407000, Ariel, Israel

${ }^{b}$ Department of Chemistry and Biochemistry, University of Hull, Hull HU6 7RX, UK

${ }^{c}$ Department of Applied Chemistry, Faculty of Engineering, Osaka Institute of Technology,

5-16-1 Omiya, Asahi-ku, Osaka 535-8585, Japan

${ }^{d}$ Nanomaterials Microdevices Research Center, Osaka Institute of Technology

5-16-1 Omiya, Asahi-ku, Osaka 535-8585, Japan

eIndustrial Engineering and Management Department, Engineering Faculty, Ariel University, P.O.B. 3, 40700, Ariel, Israel

*Corresponding author: edward@,ariel.ac.il

Paper contains ESI (10 movies) 


\begin{abstract}
A model macroscopic system imitating entering of viruses into living cells is suggested. The system represents the contact of a composite (core-shell) liquid marble with hydrophobic/hydrophilic particles. Composite liquid marbles are water droplets coated with silicone oil armored with nanometric hydrophobic particles and served as an interfacial model of a living cell. Composite marbles swallowed hydrophilic polymer particles, but prevented hydrophobic particles from entering their core. Swallowing of hydrophilic particles by composite marbles resembles the budding of living cells by viruses. The interfacial mechanism of swallowing is suggested.
\end{abstract}

Keywords: bi-liquid composite marbles; fumed fluorosilica particles; oil coating; enveloped viruses; coronavirus; budding.

Viruses are the most abundant biological entities on earth, more than single-celled organisms and bacteria together. ${ }^{1}$ They also represent the largest and most genetically diverse reservoir of nucleic acid that can infect all forms of life. Currently,1.67 million unknown viruses are estimated to be circulating in animal reservoirs, demonstrating the potential for epidemics. ${ }^{1}$ Coronavirus belongs to the so-called enveloped viruses..$^{2-4}$ Enveloped viruses have a lipid-based membrane surrounding the protein capsid. ${ }^{2-4}$ This envelope is partly composed of the cell membrane within which the virus replicated, and it contains proteins and carbohydrates. ${ }^{4}$ Viruses are tiny objects with a characteristic dimension of $c a .100 \mathrm{~nm} .^{1}$ Thus, their experimental 
exploration is extremely challenging. In particular, the experimental study of the physics of the process of viral shedding occurring via viral budding ${ }^{5}$ is a complicated task.

We suggest a macroscopic system imitating viral budding by living cells, namely we explored swallowing of solid particles by bi-liquid composite marbles. Liquid marbles are non-stick water droplets coated with nano- or micron-sized particles. ${ }^{6-8}$ Liquid marbles are not hermetically coated by solid particles. The respirability of liquid marbles makes them suitable for the cultivation of microorganisms and cells. ${ }^{9-12}$ Liquid marbles may be used as mini-reactors and bio-reactors. ${ }^{913-14}$ Use of liquid marbles for non-traditional computing was reported recently. ${ }^{15-16}$ Liquid marbles may be actuated by UV and IR $\operatorname{light}^{17}$ and Marangoni flows. ${ }^{18-19}$ Liquid marbles demonstrate obvious potential for micro-fluidics applications.. ${ }^{20-21}$ Bi-liquid, core-shell or composite marbles, i.e. water marbles coated with a thin layer of oil comprising solid hydrophobic particles, were introduced recently. ${ }^{22-24}$ The structure of the external interface of a composite liquid marble resembles that of living cell membranes, built of lipid bilayers (including cholesterols) and containing intrusions of globular proteins and fragments of the cytoskeleton, as depicted in Figure $\mathbf{1}$ a-b. In both of the composite marbles and living cells water is enveloped by a hydrophobic layer filled by heterogeneities. We do not exaggerate the physical similarity of the composite marbles and living cells, however we demonstrate that the core-shell marbles enable us to exemplify the budding of enveloped viruses within a macroscopic system. Thus, composite marbles supply an interfacial model of a living cell.

We studied the contact of composite marbles with four kinds of solid object of two different millimetrically-scaled sizes, namely: hydrophobic particles, hydrophilic particles, 
hydrophobic and hydrophilic particles coated with oil. Three very different scenarios of contact were observed: (i) pristine hydrophobic and oil-coated hydrophobic particles were attached to the surface of the liquid marble, (ii) hydrophilic particles were swallowed by the composite marbles and penetrated its core, at occurs when a living cell "absorbs" the enveloped virus; (iii) oil-coated hydrophilic particles accomplished the coating layer of the composite marbles, becoming attached to the marble in the nearest vicinity of its surface. All of these scenarios will be addressed below in detail. We pose a following general question: how do soft objects filled with water and coated with armored oil layers contact with entities possessing different hydrophilicity?

Composite marbles prepared as shown in Figure 2 and Movie S1 and described in detail in the Method section were transferred to hydrophobized glass slides. An image of the composite marble is shown in Figure 3. Objects possessing very different wetting properties (in other words various surface energies) were placed in the vicinity of the composite marble, as shown in Figure 4 and pushed gently with a spatula towards the surface of it. Let us start with the millimetre-sized hydrophobic (polypropylene, PP) particles which were attached to the surface of the composite marble, as shown in Figures 4a, 5a and Movie S2. Let us introduce the spatial scales, inherent for this experimental situation, according to Eq.1:

$$
\frac{R}{a} \gg 1 ; \frac{a}{b} \gg 1,
$$

where $R \cong 4.0 \mathrm{~mm}, a \cong 0.5 \mathrm{~mm}$ and $b \cong 50 \mathrm{~nm}$ primary diameter more like $30 \mathrm{~nm}$ are the radii of the marble, PP and fumed fluorosilica particles respectively (see Figure 1). Thus, 
hydrophobic PP particles are small relative to the marble but large relative to the fumed flurosilica particles. For the purposes of brevity we label these PP particles as "small" ones, and the fumed flurosilica particles as the "colloidal" ones. The same spatial inter-relation generally keeps for "budding" of living cells by viruses, if $R, a$ and $b$ represent the characteristic dimensions of a living cell, virus particles and globular proteins respectively (see Figure 1).

The experimental findings evidence that the small hydrophobic PP particles behave as colloidal ones and do not penetrate into the marble volume, instead adhering to the composite marble surface. The physics of this attachment was explained in refs. 6-8, when a smooth spherical particle of radius $r$ comes to the liquid surface from air, the energy gain is given by:

$$
\Delta G_{\text {air } \rightarrow \text { inteface }}=-\pi r^{2} \gamma\left(1+\cos \theta_{Y}\right)^{2}
$$

where $\theta_{Y}$ is the Young contact angle inherent to the particle/liquid/air system and $\gamma$ is the surface tension of the liquid/air interface. It is seen from Eq.2, that the entire system containing the marble and particle lowers its energy by the particle adhering to the interface regardless of the radius of the particle. However, it is thermodynamically unfavorable for the hydrophobic PP particle to enter into the water volume, and it remains attached the composite marble surface as depicted in Figures 4a, $5 \mathbf{a}$.

The behavior of hydrophilic (plasma treated) PP particles contacting the composite marble was rather different. The marble swallowed the particle as shown in Figures $\mathbf{4 b}, \mathbf{5 b}$ and Movie S4, S5. Hydrophilic particles penetrated into the core of the composite marble, thus 
resembling the behavior of enveloped viruses under their budding of the living cell, as shown in Figures 4b, 5b and Movie S4. The characteristic time of the "swallowing" was established experimentally as $\tau \cong ? ? ?$ PRITAM. The mechanism of the "swallowing" is suggested in Figure 6: interface with an area $S$ separates a hydrophilic particle from water. The particle is hydrophilic, thus wetting of this surface is thermodynamically favorable and results in the free energy gain:

$$
\Delta \Phi=\left(\gamma_{s / w}-\gamma_{s / a}\right) S=-\gamma S \cos \theta_{Y}
$$

where $\gamma_{s / w}$ and $\gamma_{s / a}$ are the interfacial tensions at the solid/water and at the bare solid/air interfaces, respectively. The free energy gain $\Delta \Phi$ is increased with the increase of the wetted area $S$. Thus, the force $|\vec{F}|=\left|\frac{\partial \Phi}{\partial r}\right|$ (where $r$ is the radial coordinate of the center of mass of the hydrophilic particle) emerges, pushing the particle into the water core (see Figure 6). The force $\vec{F}$ acts until the wetted area is increased, in other words until complete wetting of a hydrophilic particle by water. It seems reasonable to suggest that the similar interfacial mechanism governs entering of viruses into living cells. However, the study of the exact mechanism of virus budding of living cells remains a challenging experimental and theoretical task. It is noteworthy that the study of our model system only sheds light on the interfacial aspect of this mechanism.

In the case of oil-coated hydrophobic and hydrophilic particles the wetting scenario was different. Both of these particle types adjusted themselves to the coating layer of liquid marbles, thus accomplishing wrong word- not sure what you are trying to say? it as demonstrated in 
Figure 4c-d and Movies S6, S7. It was observed that oil-coated hydrophobic particles are attached to the composite marble surface outside of the marble, whereas oil-coated hydrophilic particles are adhered to the composite marble, keeping the PP particles connected to the oil layer from inside.

We also studied the contact of large $a \approx 2.5 \mathrm{~mm}$ hydrophobic and plasma hydrophilized PP particles by the composite marble, illustrated in Figures 6a, b and $\mathbf{c}, \mathbf{d}$ and the supporting Movies S8, S9 and S10. In this case the spatial scales are given by Eq. 4:

$$
\frac{R}{a} \cong 1 ; \frac{a}{b} \gg 1
$$

The nature of the interfacial processes remained the same irrespective of the change in the spatial scales, namely: hydrophobic PP polymer particles did not penetrate into the composite marble core, whereas plasma hydrophilized PP particles were swallowed by the composite marble, as shown in Figure 7. The characteristic time of the "swallowing" was established experimentally as $\tau \cong ? ? ?$ PRIT AM.

We conclude that the composite (core-shell) marbles swallowed hydrophilic polymer particles, thus supplying the interfacial model of the processes occurring under budding of living cells by viruses. At the same time, the composite marbles prevented hydrophobic particles from entering their core. This conclusion is true for particles of various sizes.

\section{Materials and Methods}




\section{Materials}

The following materials were used in order to prepare the sandwich liquid marbles make full sentences:

- microscope glass slides $(3 \mathrm{~mm} \times 2 \mathrm{~mm})$;

- $\quad$ poly(dimethylsiloxane) (PDMS) Sylgard 184, supplied by Dow Corning, USA;

- deionized water (DI) from Millipore SAS (France) (specific resistivity $\hat{\rho}=18.2 \mathrm{M} \Omega \mathrm{cm}$ at $25^{\circ} \mathrm{C}$, surface tension $\gamma=72.9 \mathrm{mN} / \mathrm{m}$; viscosity $\eta=8.9 \times 10^{-4} \mathrm{Pas}$ );

- Poly(dimethylsiloxane), bis(hydroxyalkyl) terminated supplied by Sigma-Aldrich (molecular weight $5600 \mathrm{~g} / \mathrm{mol}$, surface tension $\gamma=19.2 \mathrm{mN} / \mathrm{m}$, viscosity $\eta=0.1 \mathrm{Pas}$ (100 cSt), volume magnetic susceptibility $\chi_{\text {oil }}=-7.5 \times 10^{-6}$ not needed?, boiling point $449.7 \pm 45.0^{\circ} \mathrm{C}$ at $\left.760 \mathrm{mmHg}\right)$;

- fumed fluorosilica particles were synthesized according to the protocol reported in ref. 26; the primary particle diameter is approx. $30 \mathrm{~nm}$.

A Nikon 1 v3 camera was used to capture images, movies of composite liquid marbles and the process of swallowing of different particles.

Polypropylene (PP) particles of two diameters (1 $\mathrm{mm}$ and $5 \mathrm{~mm})$, supplied by Sigma-Aldrich, were used as hydrophobic particles. The apparent water contact angle of pristine PP was $\theta=? ? ?$ Pritam

\section{Methods}


Hydrophobic glass substrates were prepared by coating of the cold plasma-treated microscope glass slides with a very thin layer of PDMS oil as described in our earlier work [35]. The slides were coated with silicone oil with a spin coater (Laurell Technologies, WS-650MZ-23NPPB Spin Processor) at $5000 \mathrm{rpm}$. A $5 \mu \mathrm{L}$ water drop was deposited using a micropipette on the oil-coated hydrophobic glass. The spreading parameter describing wetting of the silicone oil/water system is positive (for the detailed analysis of the spreading parameter see the discussion in ref. 25); thus, the silicone oil covers the water drop. Tilting of the glass substrate forced the water drop covered with silicone oil to roll to the Petri dish containing fumed fluorosilica particles, as shown in Figure 2. The particles adhere to the drop giving rise to a stable composite liquid marble as depicted in Figure 3. The process of composite liquid marble preparation, carried out under ambient conditions, is illustrated in the supplementary Movie S1. The weight ratio of the components of the composite marbles, namely water/oil/particles was established by weighing as $1 / 0.054 / 0.102$.

Polypropylene (PP) particles were plasma treated using an inductive radio frequency air plasma discharge with the following parameters: frequency was $13.56 \mathrm{MHz}$; air pressure was $0.5 \mathrm{Torr}$; power of the plasma discharge was $18 \mathrm{~W}$; time span of irradiation was $300 \mathrm{~s}$. The apparent water contact angle of plasma treated PP was $\theta=$ ??? Pritam.

Apparent contact angles were measured with a Rame-Hart goniometer at ambient conditions.

\section{References}


1. Yeh, Y. T.; Gulino, K.; Zhang, Y.; Sabestien, A.; Chou, T. W.; Zhou, B.; Lin, Z.; Albert, I.;Lu, H.; Swaminathan, V.; Ghedin, E. A rapid and label-free platform for virus capture and identification from clinical samples. PNAS 2020, 117, 895-901.

2. Weissenhorn, W.; Dessen, A.; Calder, L. J.; Harrison, S. C.; Skehel, J. J.; Wiley, D. C. Structural basis for membrane fusion by enveloped viruses. Mol. Membr. Biol. 1999, 16, 3-9.

3. White, J. M.; \& Whittaker, G. R. Fusion of enveloped viruses in endosomes. Traffic 2016, 17, 593-614.

4. Fenouillet, E.; Barbouche, R.; Jones, I. M. Cell Entry by Enveloped Viruses: Redox Considerations for HIV and SARS-Coronavirus. Antioxid. Redox Signal. 2007, 9, 1009-1034.

5. Pornillos, O.; Garrus, J. E.; Sundquist, W. I. Mechanisms of enveloped RNA virus budding. Trends Cell Biol. 2002, 12, 569-79.

6. Aussillous, P.; Quéré, D. Liquid marbles, Nature 2001, 411, 924-927.

7. Li, X. Liquid marbles and liquid plasticines with nanoparticle monolayers. $A d v$. Colloid Interface Sci. 2019, 271, 101988.

8. Bormashenko, E. Liquid marbles, elastic nonstick droplets: from minireactors to self-propulsion. Langmuir 2017, 33, 663-669.

9. Arbatan, T.; Li, L.; Tian, J.; Shen, W. Liquid marbles as micro-bioreactors for rapid blood typing. Adv. Healthc. Mater. 2012, 1, 80-83. 
10. Tian, J.; Fu, N.; Chen, X. D.; Shen, W. Respirable liquid marble for the cultivation of microorganisms. Colloids Surf. B 2013, 106, 187-190.

11. Serrano, M. C.; Nardecchia, S.; Gutiérrez, M. C.; Ferrer, M. L.; del Monte, F. Mammalian cell cryopreservation by using liquid marbles, ACS Appl. Mater. Interfaces 2015, 7, 3854-3860.

12. Vadivelu, R. K.; Ooi, Ch. H.; Yao, R.-Q.; Velasquez, J. T.; Pastrana, E.; Nido, J. D.; Lim, F.; Ekberg, J. A. K.; Nguyen, N-Tr.; St John, J. A. Generation of three-dimensional multiple spheroid model of olfactory ensheathing cells using floating liquid marbles, Sci. Rep. 2015, 5, 1508.

13. Fullarton, C.; Draper, T. C.; Phillips, N.; de L. Costello, B. P J; Adamatzky, A.; Belousov-Zhabotinsky reaction in liquid marbles. J. Phys.: Mater 2019, 2, 015005.

14. Adamatzky, A.; Fullarton, C.; Phillips, N.; De Lacy Costello, B.; Draper, T. C. Thermal switch of oscillation frequency in Belousov-Zhabotinsky liquid marbles. $R$. Soc. Open Sci. 2019, 6, 190078.

15. Fullarton, C.; Draper, T. C.; Phillips, N.; Mayne, R.; de Lacy Costello, B. P.; Adamatzky, A. Evaporation, Lifetime, and Robustness Studies of Liquid Marbles for Collision-Based Computing. Langmuir 2018, 34, 2573-2580.

16. Draper, T. C.; Fullarton, C.; Phillips, N.; de Lacy Costello, B. P.; Adamatzky, A. Liquid marble interaction gate for collision-based computing, Mater. Today 2017, 20, 561-568. 
17. Paven, M.; Mayama, H.; Sekido, T.; Butt, H. J.; Nakamura, Y.; Fujii, S. Light-driven de-livery and release of materials using liquid marbles, Adv. Funct. Mater. 2016, 26, 3199-3206.

18. Fujii, S.; Yusa, S. I.; Nakamura, Y. Stimuli-responsive liquid marbles: controlling structure, shape, stability and motion, Adv. Funct. Mater. 2016, 26, 7206-7223.

19. Bormashenko, E.; Bormashenko, Y.; Grynyov, R.; Aharoni, H.; Whyman, G.; Binks, B. P. Self-propulsion of liquid marbles: Leidenfrost-like levitation driven by Marangoni flow. J. Phys. Chem. C 2015, 119, 9910-9915.

20. Ooi, C. H.; Jin, J.; Nguyen, A. V.; Evans, G. M.; Nguyen, N. T. Picking up and placing a liquid marble using dielectrophoresis. Microfluid. Nanofluidics 2018, 22, 142.

21. Jin, J.; Ooi, C. H.; Sreejith, K. R.; Dao, D. V.; Nguyen, N-Tr. Dielectrophoretic trapping of a floating liquid marble. Phys. Rev. Appl. 2019, 11, 044059.

22. Takei, T.; Yamasaki, Y.; Yuji, Y.; Sakoguchi, S.; Ohzuno, Y.; Hayase, G.; Yoshida, M. Millimeter-sized capsules prepared using liquid marbles: Encapsulation of ingredients with high efficiency and preparation of spherical core-shell capsules with highly uniform shell thickness using centrifugal force, J. Colloid and Interface Sci. 2019, 536, 414-423.

23. Sreejith, K. R.; Gorgannezhad, L.; Jin, J.; Ooi, C. H.; Takei, T.; Hayase, G.; Stratton, H.; Lamb, K.; Shiddiky, M.; Dao, D. V.; Nguyen, N-T. Core-shell beads made by composite liquid marble technology as a versatile microreactor for polymerase chain reaction, Micromachines 2020, 11, 242. 
24. Roy, P. K.; Binks, B. P.; Bormashenko, Ed.; Legchenkova, I.; Fujii, S.; Shoval, Sh. Manufacture and properties of composite liquid marbles, J. Colloid and Interface Sci. 2020, https://doi.org/10.1016/j.jcis.2020.04.066

25. Multanen, V.; Chaniel, Ch.; Grynyov, R.; Loew, R. Y.; Siany, N. K.; Bormashenko, E. Hydrophilization of liquid surfaces by plasma treatment, Colloids Surf. A 2014, 461, 225-230.

26. Binks, B. P.; Tyowua, A. T. Influence of the degree of fluorination on the behavior of silica particles at air-oil surfaces. Soft Matter 2013, 9, 834-845.
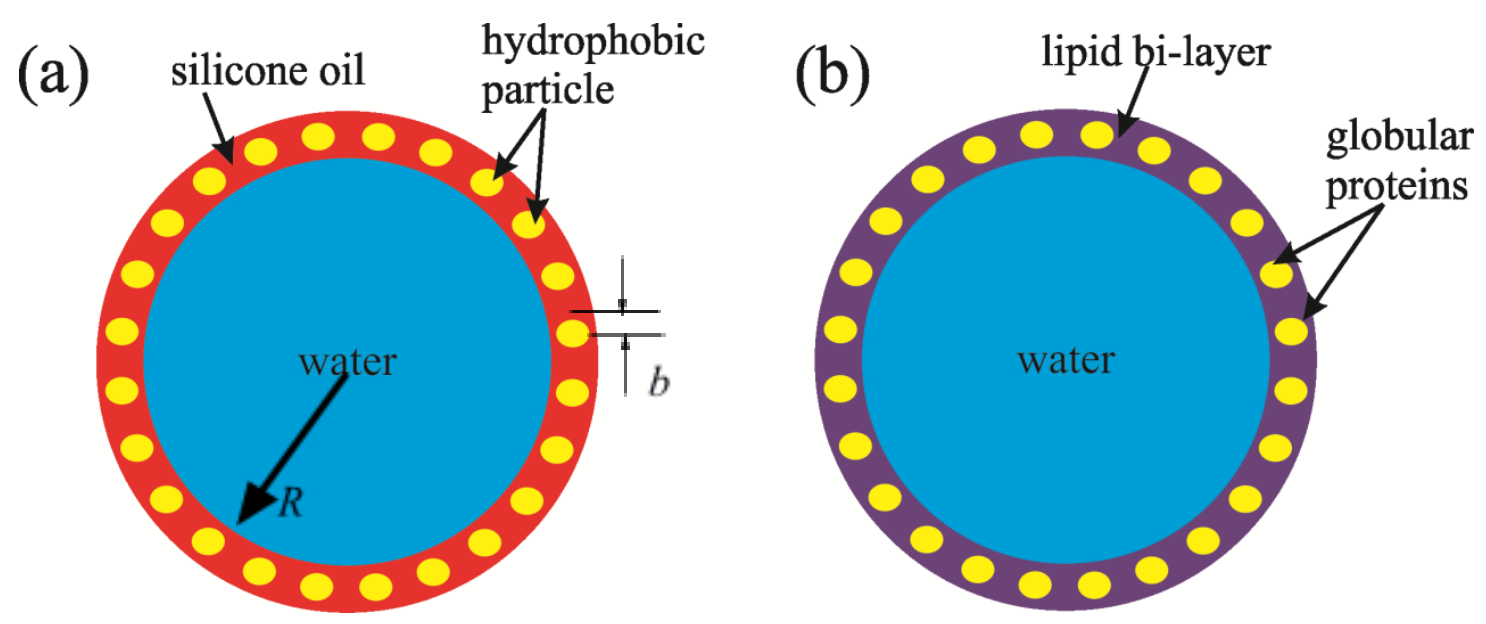
Figure 1. Sketch demonstrating the interfacial resemblance of a composite liquid marble (a) and living cells (b); the radius of the marble $R \cong 4.0 \mathrm{~mm}$; the primary diameter of the hydrophobic fumed fluorosilica particles $b \cong 30 \mathrm{~nm}$. 


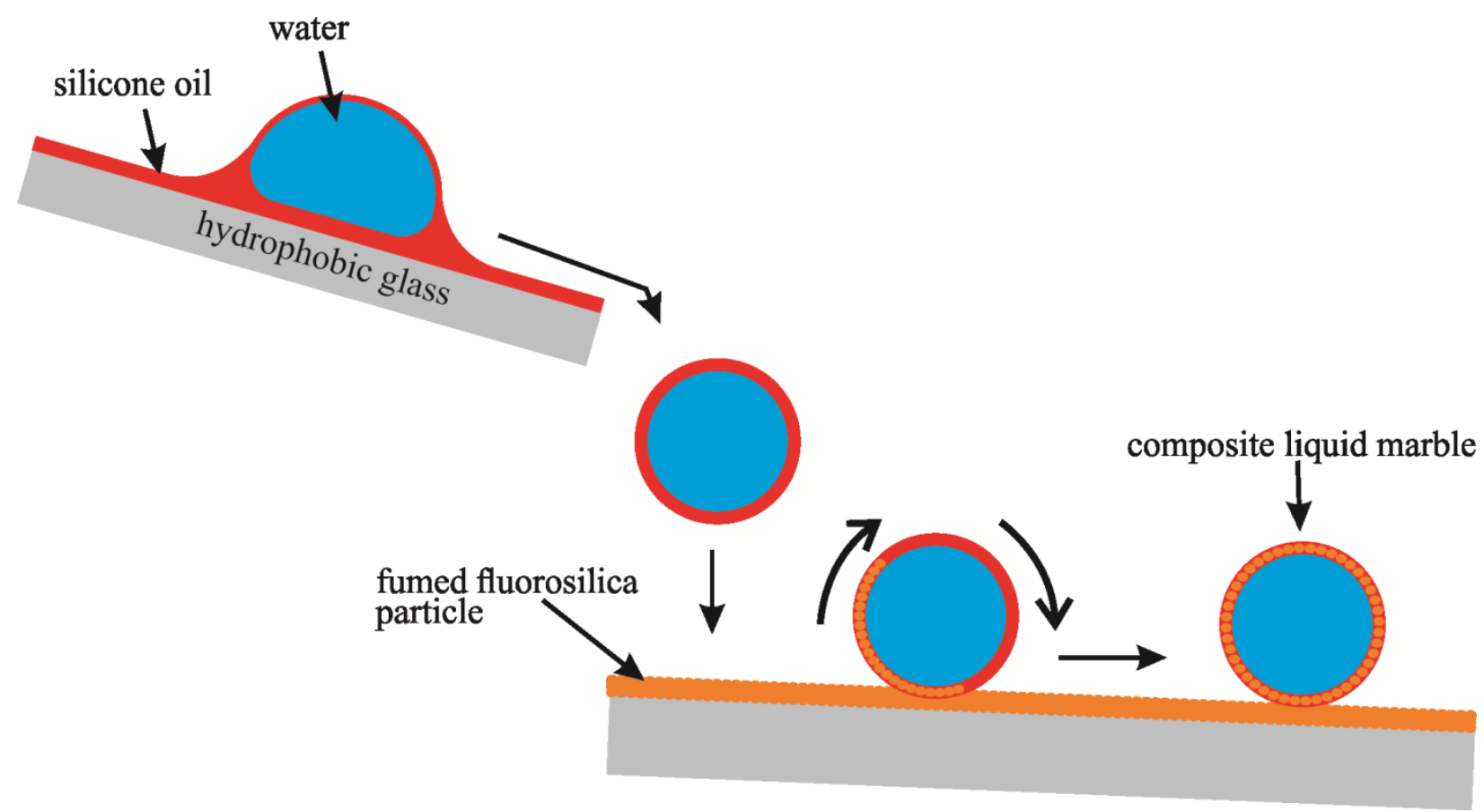

Figure 2. Preparation of a composite liquid marble is shown. A water droplet coated with silicone oil rolls along a hydrophobic substrate and falls onto a solid substrate containing fumed fluorosilica particles. The process results in the formation of a composite (core-shell) marble. 


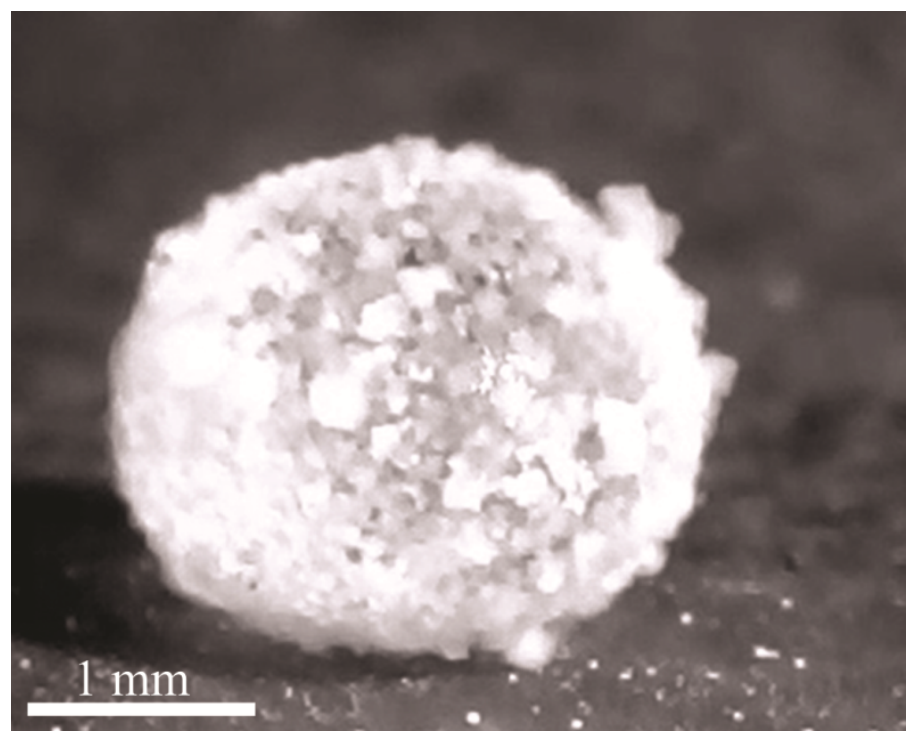

Figure 3. Optical image of a composite liquid marble ( $V=? ?$ ? Pritam) is presented. 

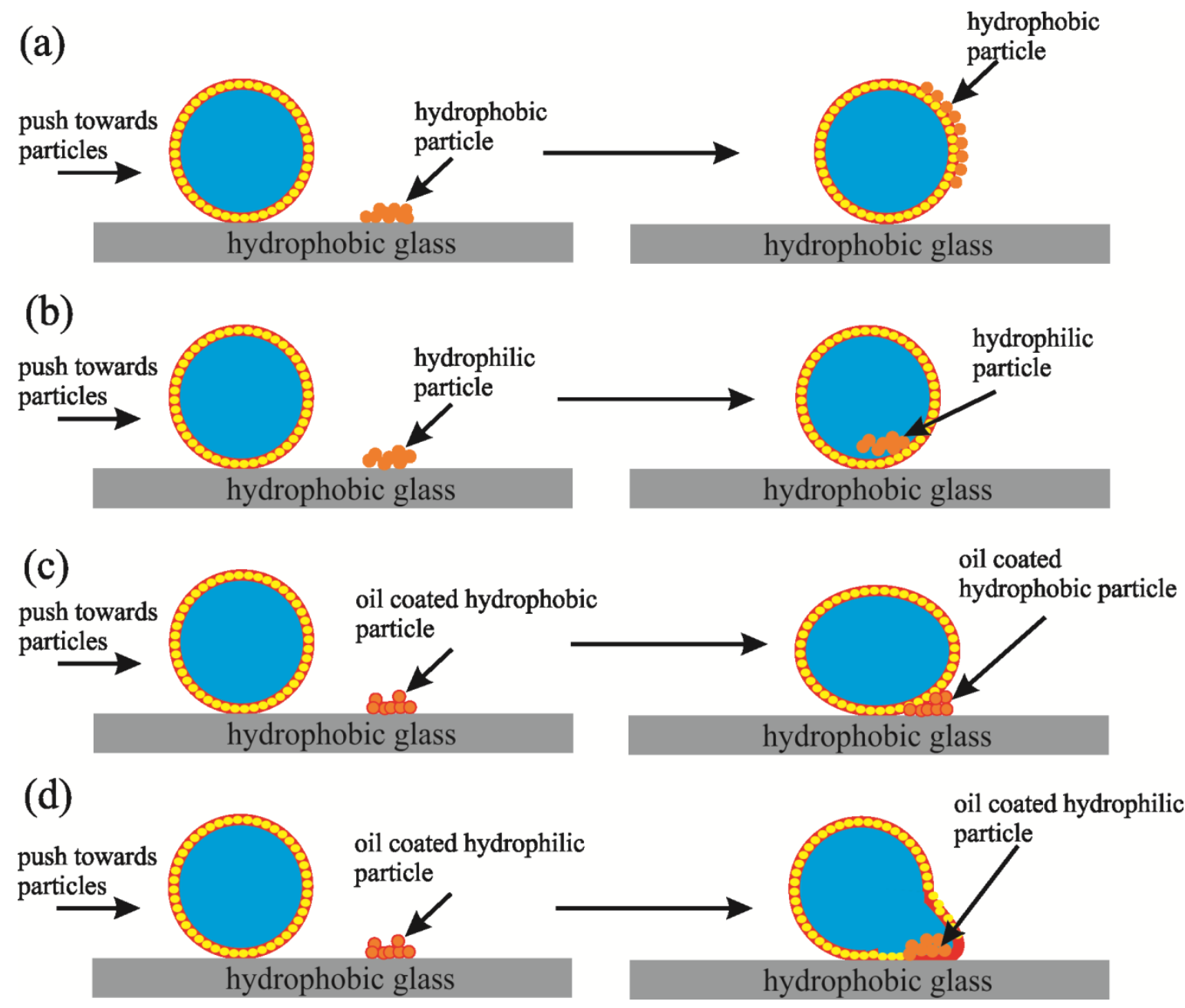

Figure 4. Scheme illustrating the contact between hydrophobic (a), hydrophilic (b), oil-coated hydrophobic (c) and oil-coated hydrophilic (d) particles with a composite liquid marble. 


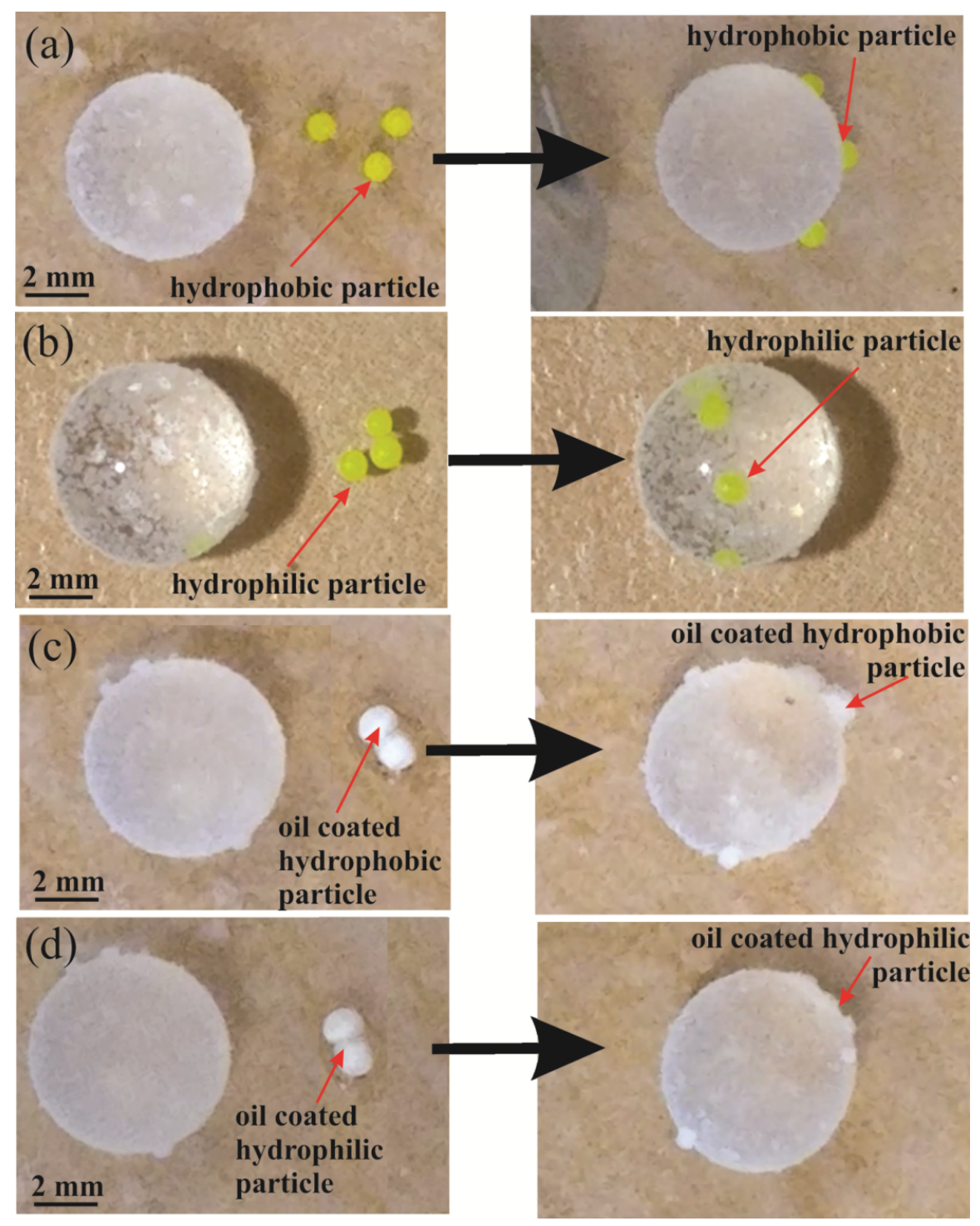

Figure 5. Optical images show contact between hydrophobic (a), hydrophilic (b), oil-coated hydrophobic (c) and oil-coated hydrophilic (d) particles with a composite liquid marble. in (b) the original marbles appears to have bare patches on its surface: is this why particles enter? in (b), how can you prove that particles are actually in the core water? some could argue they are on the surface... 


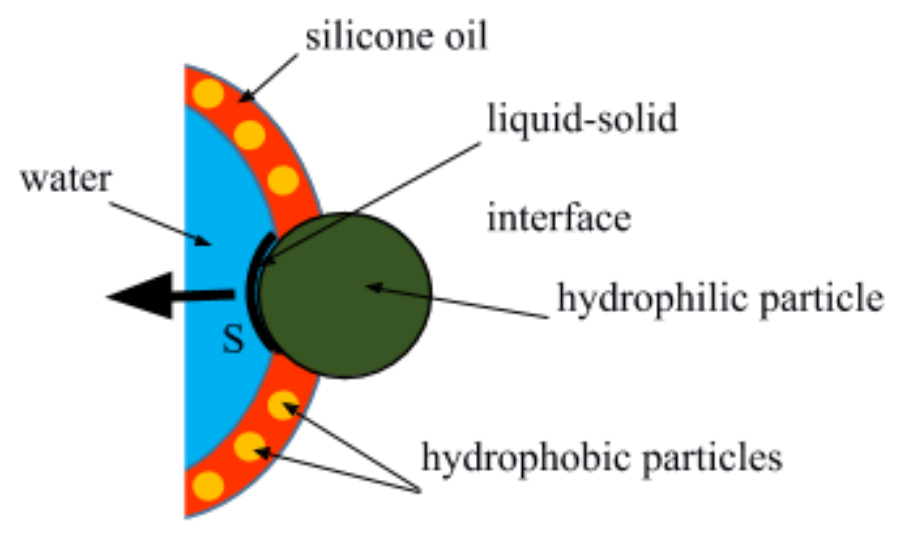

Figure 6. Mechanism of penetration of hydrophilic particles into the bulk of a composite liquid marble. Interface of area $S$ separates a hydrophilic particle from water. Force $\vec{F}$ pushes particle? into the water core. 

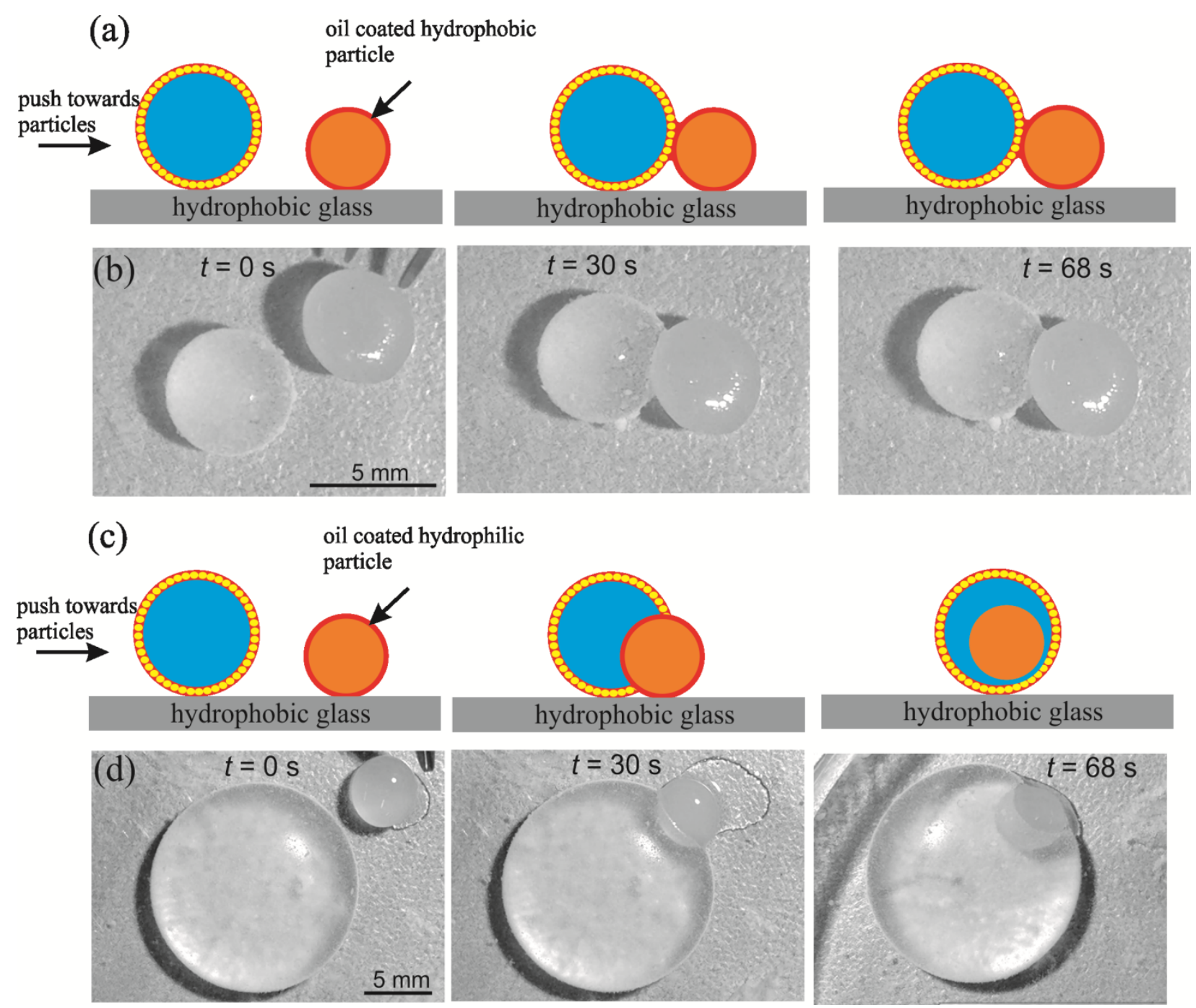

Figure 7. Attachment of oil-coated hydrophobic particle to a composite liquid marble: (a) scheme, (b) sequence of images illustrating particle attachment. Swallowing of oil-coated hydrophilic polystyrene particle by a composite liquid marble: (c) scheme, (d) sequence of images representing the swallowing. 


\section{TOC image}
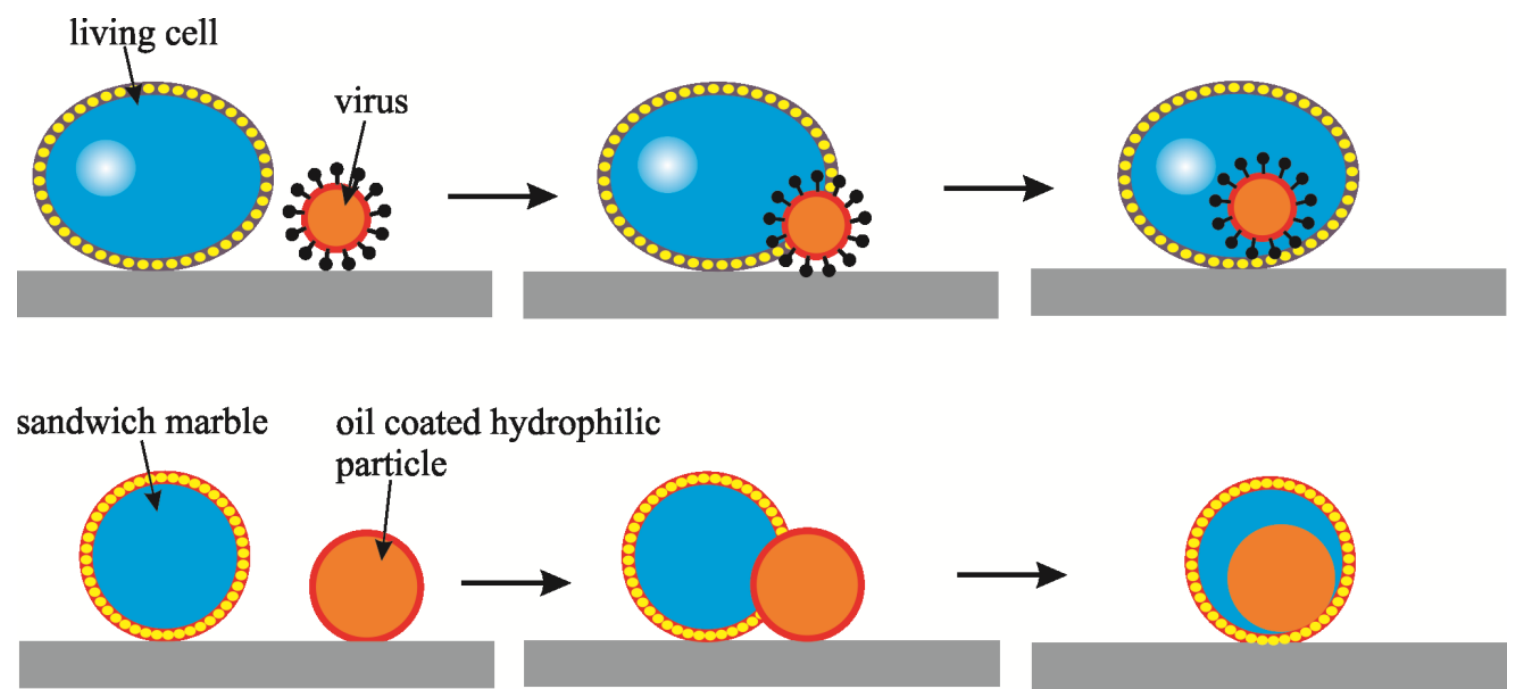

change 'sandwich' to composite 